M. D. Smith

Astronomy Program, University of Maryland and

J. R. Dicke1

Department of Astronomy, University of Illinois.

ABSTRACT. Rapid cooling and collapse in a thermally unstable medium can lead to filamentary structures that are comparable to those observed in old supernova remnants. Here, simple formulae are derived for the shape of a filament forming in this manner. The cross-sectional shape of a filament will flatten and the ratio of major to minor axes (aspect ratio) increases in proportion to the density as it becomes sheet-like. Thus the aspect ratio of observed filaments is expected to be of the same order as the temperature change during the collapse, $\mathrm{T}_{1} / \mathrm{T}_{0}$. In very old remnants the filaments may appear to be tubes or sheets depending on perspective with aspect ratios less than 10 . This model predicts a correlation between optical morpholggy and the temperature of the shock-heated gas. For $\mathrm{T}_{1}$ between $10^{5} \mathrm{~K}$ and $10^{6} \mathrm{~K}$, the thin sheet that forms is likely to split up through asymmetric warping disturbances, leading to several parallel stfiations, consistent with observations in the Cygnus Loop. Above $10^{6} \mathrm{~K}$, the instabifity may cause the sheet to degenerate before it has cooled to $10^{4} \mathrm{~K}$. Hence, an absence of we11defined filaments is expected near $X$-ray emitting regions.

1. THE COLLAPSE

As a region of gas cools behind a shock front, it can collapse and fragment. These collapsed regions are thought to correspond to observed optical filaments in old supernova remnants. Thermal instabilities cause the fragmentation. That is, regions of greater density cool faster than regions of lower density, and thus, to keep in pressure balance, become even denser. They will apply to regions in which the sound crossing time, $t_{s}$, is shorter than the cooling time, $t_{c} \cdot$ Using a mathematical technique involving a small parameter called the slenderness ratio, $\varepsilon=t_{s} / t$, it is possible to accurately follow the collapse in two dimensions (Smith and Dickel 1983).

The results obtained by the above method are easily generalized. This is best seen by taking an ellipsoid with axes $a, b$ and $c$, and external pressure $P_{e}$ and central pressure $P_{c}{ }^{\cdot}$ As a result 
of radiative cooling within the ellipsoid the central pressure is slightly less than the external pressure. It is this small pressure difference which drives the collapse. The rates of change of the lengths of the axes depends on the pressure gradients $\left(\mathrm{P}_{e^{-}}^{\left.-P_{c}\right) / a,}\left(\mathrm{P}_{e^{-}}\right.\right.$ $\left.P_{c}\right) / b$ and $\left(P_{e}-P_{c}\right) / c$. Since the pressure gradient is greatest along the shortest axis, the collapse is mainly in this direction. Thus, the ellipsoid flattens and once, say, $a \ll b, c$ the collapse is essentially only along the a-axis.

With the collapse, the magnetic field becomes more ordered. Thermal conduction inhibits temperature variations in the field direction and we can then consider a two dimensional collapse. The cross-sectional area of a filament is inversely proportional to the mass density (mass conservation) and, further, is proportonal to the temperature provided the external pressure remains unchanged. It follows that the axial ratio is given by

$$
\begin{aligned}
& a / b \sim 0\left(T_{i} / T_{f}\right) \text { for } a>b, \text { and } \\
& a / b \propto T_{i} / T_{f} \text { for } a \gg b
\end{aligned}
$$

where $T_{f}$ and $T_{f}$ are the initial and $f$ inal temperatures of the collapsing region.

Through the slenderness method we find that a sheet is unstable to warping. Initial irregularities in the shape of a region are preserved in size while the region collapses. Thus irregularities are amplified relative to the sheet thickness. Given that the initial shape is far from elliptical, the instability will split up the filament. We do not expect a thin sheet to survive intact for very long.

\section{OBSERVATIONAL IMPLICATIONS}

Putting $\mathrm{T}_{\mathrm{f}} \sim 10^{4} \mathrm{~K}$, we can find the structure at the optically observable stage. With $\mathrm{T}_{i} \leqslant 10^{5} \mathrm{~K}$ we do not expect a great collapse or change in shape. For $10^{6} \mathrm{~K}>\mathrm{T}_{1}>2.10^{5} \mathrm{~K}$ we expect several parallel striations in the form of sheets wiht axial ratios of $10: 1$. For $\mathrm{T}_{1}>10^{6} \mathrm{~K}$, highly unstable thin sheets are predicted, resulting in more diffuse filamentary structures. Hence, even though there is no observed correlation between $X$-ray and optical brightness distributions, we predict a correlation between $X$-ray emission and the optical structure. A further test of the model would be to determine the depth of filaments as done by Miller (1974), who concluded that certain filaments in the Cygnus Loop are sheets.

As J. Dickel has remarked in this Symposium, much work still needs to be done in this area. The full story must include thermal conduction, inertial collapse and magnetic field effects. We encourage observations of individual filaments in both the radio and optical. This will lead to tighter constraints on physical parameters and give the theoretician a more precise task. 


\section{REFERENCES}

Miller, J.S. 1974, Ap. J., 189, 239.

Smith, M. D., and Dickel, J. R. 1983, Ap. J. February 1.

\section{DISCUSSION}

DANZIGER: I performed a similar excercise as Miller (on Cygnus) for a filament in the Vela supernova remnant, and obtained a similar result, viz. a length in the line of sight about an order of magnitude greater than the observed width.

BENFORD: How much does the presence of a significant embedded magnetic field - which will be compressed and perhaps amplified affect your results?

SMITH: One must first determine the strength of the magnetic field in the interstellar medium. In cool clouds the field and thermal gas may be in approximate pressure balance, whereas the thermal pressure may dominate in the hot intercloud gas. In either case, the compression and heating by a blast wave leaves the magnetic pressure relatively small. In the subsequent collapse, the magnetic field within the region rapidly increases. For high $\mathrm{T}_{1}$ the magnetic pressure can balance the external pressure before the filament has cooled to $10^{4} \mathrm{~K}$. At this stage, however, the filament is likely to be collapsing inertially i.e. ahead of pressure equilibrium, and hence, the collapse towards a sheet continues. Finally it should be noted that it is difficult to make dense filaments if the field pressure or its turbulent amplification is of greater significance. 